\title{
Intrinsic and extrinsic resistive switching in a planar diode based on silver oxide nanoparticles
}

\author{
Asal Kiazadeh a , Henrique L. Gomes ${ }^{\text {a,* }}$, Ana M. Rosa da Costa b , José A. Moreira ${ }^{\text {b }}$, \\ Dago M. de Leeuw ${ }^{c}$, Stefan C.J. Meskers ${ }^{\mathrm{d}}$ \\ a Instituto de Telecomunicações, Universidade do Algarve, Campus de Gambelas, 8005-139 Faro, Portugal \\ b Centro de Investigação em Química do Algarve, Universidade do Algarve, Campus de Gambelas, 8005-139 Faro, Portugal \\ ' Philips Research Laboratories, High Tech Campus 4 WAG 11, 5656 AE Eindhoven, The Netherlands \\ d Molecular Materials and Nanosystems, Eindhoven University of Technology, P.O. Box 513, 5600 MB Eindhoven; The Netherlands
}

\section{A R T I C L E I N F O}

\section{Article history:}

Received 4 January 2012

Received in revised form 14 August 2012

Accepted 17 August 2012

Available online 30 August 2012

\section{Keywords:}

Nanoparticles

Resistive switching

Non-volatile memory

\begin{abstract}
A B S T R A C T
Resistive switching is investigated in thin-film planar diodes using silver oxide nanoparticles capped in a polymer. The conduction channel is directly exposed to the ambient atmosphere. Two types of switching are observed. In air, the hysteresis loop in the current-voltage characteristics is S-shaped. The high conductance state is volatile and unreliable. The switching is mediated by moisture and electrochemistry. In vacuum, the hysteresis loops are symmetric, $\mathrm{N}$-shaped and exhibit a negative differential resistance region. The conductance states are non-volatile with good data retention, programming cycling endurance and large current modulation ratio. The switching is attributed to electroforming of silver oxide clusters.
\end{abstract}

(c) 2012 Elsevier B.V. All rights reserved.

\section{Introduction}

Resistive switching is a phenomenon by which a material can undergo reversible changes in electrical resistance. There is a world-wide effort to elucidate the underlying physics and to develop a resistive random access memory. Prototype memories are all based on metalinsulator-metal (MIM) diodes, but are distinguished by the nature of the switching material that range from polymers through organic molecules to blends incorporating dyes, salts, and conducting nanoparticles [1-4]. Apart from the diversity of materials there is also a variety of current-voltage $(I-V)$ characteristics. Recently, Scott et al. [5] and Prime and Paul [6] have reviewed the most common types of $I-V$ characteristics. Interesting ones have been referred to as S-shaped and N-shaped $I-V$ curves [7-11]. Both demonstrate a low current of up to a threshold voltage where a sudden increase in current occurs. However, the electrical behavior to revert from the on-state to the low conductance off-state for each type is different. The S-shaped curve reverts to the off-state when the voltage is reduced to zero or when an opposite polarity voltage pulse is applied (bipolar switching). The N-shaped curve is characterized by a voltage region with a negative differential resistance (NDR) region and unipolar programming voltages.

A correlation between the shape of the $I-V$ hysteresis loops and a particular class of material or diode has not yet been established. According to literature, MIM structures based on metal nanoparticles

\footnotetext{
* Corresponding author at: Universidade do Algarve, FCT, Campus de Gambelas, 8005-139-Faro, Portugal. Tel.: +351 289800900x7763; fax: + 351289800066.

E-mail address: hgomes@ualg.pt (H.L. Gomes).
}

can show either $\mathrm{N}$-shape or S-shape hysteresis loops. For instance, for diodes incorporating Au nanoparticles, both N-shape [12,13] and S-shape $[14,15]$ hysteresis loops have been reported by various research groups. It is disconcerting that identical diodes can have either S-shape or N-shape hysteresis loops. Recently, the coexistence of bipolar resistive switching and unipolar resistive switching has also been reported [16-19]. The explanations are diverse, and some authors claim that the type of switching mechanism is dependent on the polarity of the electroforming process [20], while another study on $\mathrm{Ni}|\mathrm{NiO}| \mathrm{Ni}$ based resistive random access memories refers that the type of switching can be selected by controlling the oxidation time of the NiO thin film [16].

Here we deliberately use planar diodes that allow the investigation of switching as a function of the ambient atmosphere. We show that the same diode can exhibit both S-shaped and N-shaped I-V curves depending on the ambient atmosphere. The S-shape is observed in air while the N-shape is only observed in vacuum. Although the results do not allow a generalization of all the $\mathrm{S}$-shaped behaviors reported in literature, this finding may help clarify the role of the atmosphere in the resistive switching.

\section{Experimental details}

Silver nanoparticles were prepared by reducing silver nitrate in the presence of poly-vinyl-pyrrolidone (PVP). In a typical experiment, PVP $(0.2 \mathrm{~g})$ was dissolved in $25 \mathrm{~mL}$ dimethylformamide. An aqueous solution of silver nitrate $(0.01 \mathrm{M}, 10 \mathrm{~mL})$ was prepared and added to the PVP solution drop wise under stirring (300 rpm) in ambient atmosphere for almost $4 \mathrm{~h}$. The solution turns from orange to 
brown showing the presence of silver oxide nanoparticles. The average size $(<20 \mathrm{~nm})$ was estimated from transmission electron microscopy (TEM) using a FEG Philips CM 200 with an operating voltage of $200 \mathrm{kV}$. The colloidal solution comprising silver oxide nanoparticles in PVP was deposited on top of preformed interdigitated Au microelectrode arrays, fabricated on $1 \mu \mathrm{m}$ thermally oxidized silicon wafers (see Fig. 1). The microelectrode arrays were $10.000 \mu \mathrm{m}$ long and separated by $10 \mu \mathrm{m}$, unless specified otherwise. Thin films were formed by drop casting and pumped for several hours to remove residual solvent. Electrical measurements were carried out using a Keithley 487 picoammeter/voltage source.

\section{Results}

A typical example of an $S$-shaped $I-V$ characteristic is shown in Fig. 2(a). The diode is measured in air and starts in the off-state. At a negative voltage of $-9 \mathrm{~V}$ a sudden increase in current occurs to the high conductance on-state. With the voltage scanning back towards zero the diode remains in the on-state down to near $0 \mathrm{~V}$ where it suddenly switches back to the low conductance off-state. $I-V$ curves are symmetrical. This S-shaped $I-V$ curve is always observed when the diodes are measured in air. Variations are possible; sometimes the diode remains in the on-state even after passing through $0 \mathrm{~V}$ and only returns to the off-state after applying an opposite bias. Although this behavior resembles a bipolar type of switching, the voltage where it returns to the off-state fluctuates substantially between 0 and $-7 \mathrm{~V}$. Therefore, it suggests that the reverse voltage does not play a significant role in the conductance turn-off. The retention time of this on-state can reach two hours as shown in Fig. 2(b).

When the same diodes are measured in vacuum, the switching behavior is completely different. Initially, the diodes are insulating. A voltage ramp of up to $50 \mathrm{~V}$ has to be applied before the diode becomes a bistable switch. When compared to the $9 \mathrm{~V}$ applied in the S-shaped switching, it is clear that a substantially higher voltage is necessary to induce switching. This step corresponds to an electroforming process.
Although electroforming has been reported for a large number of systems [21], the nature of the process is still elusive. Electroforming cannot be observed in air in our planar structure. After electroforming the diode shows sharp transitions of the electrical resistance. The high conductance on-state exhibits symmetrical NDR regions located at around $|35| \mathrm{V}$ as shown in Fig. 3. The switching biases are indicated as $V_{\text {on }}$ and $V_{\text {off. }}$ A pulse near $V_{\text {off }}$, i.e. at the bottom of the NDR, will bring the diode to the lower $I-V$ curve. The high conductance $I-V$ curve is restored by using a pulse near $V_{\text {on }}$, i.e. at the top of the NDR, typically $|30| \mathrm{V}$. The on/off ratio is 3 orders of magnitude at a reading voltage of $3 \mathrm{~V}$. The switching behavior in Fig. 3 is usually referred to as the $\mathrm{N}$-shape. The states retain the programmed conductance state without bias as long as they are stored in vacuum (Fig. 4(a)). Once the diode is programmed into a certain state, it can be reliably read-out many times without degradation as shown in Fig. 4(b). Data retention is excellent.

In addition to the $I-V$ characteristics, $\mathrm{S}$-shape switching and $\mathrm{N}$-shape switching can also be compared in terms of the voltage required to program on-state and off-state. Fig. 5 shows the switching voltage dependence on channel length $(L)$. S-shape switching fluctuates for identical samples in the voltage range of $5-8 \mathrm{~V}$ and it is almost insensitive to change in channel length. For N-shape switching, the voltage changes from $15 \mathrm{~V}$ up to $140 \mathrm{~V}$ as the channel length increases from $5 \mu \mathrm{m}$ to $40 \mu \mathrm{m}$. This noticeable difference in the magnitude of the switching voltage suggests that the associated mechanisms are different.

In order to get insight into the switching mechanism, temperature dependent measurements were carried out. To change the sample temperature, vacuum conditions are required, therefore, these studies were only possible in N-shape switching. Fig. 6 shows the temperature dependence of the $I-V$ curves. As the sample is cooled down to $190 \mathrm{~K}$ the current decreases and the NDR region moves to higher voltages. The thermal activated behavior shows that this type of switching is not caused by the presence of metallic filaments.

For the diodes discussed above, the S-shape switching is only observed in air. However, it is possible to produce diodes working in
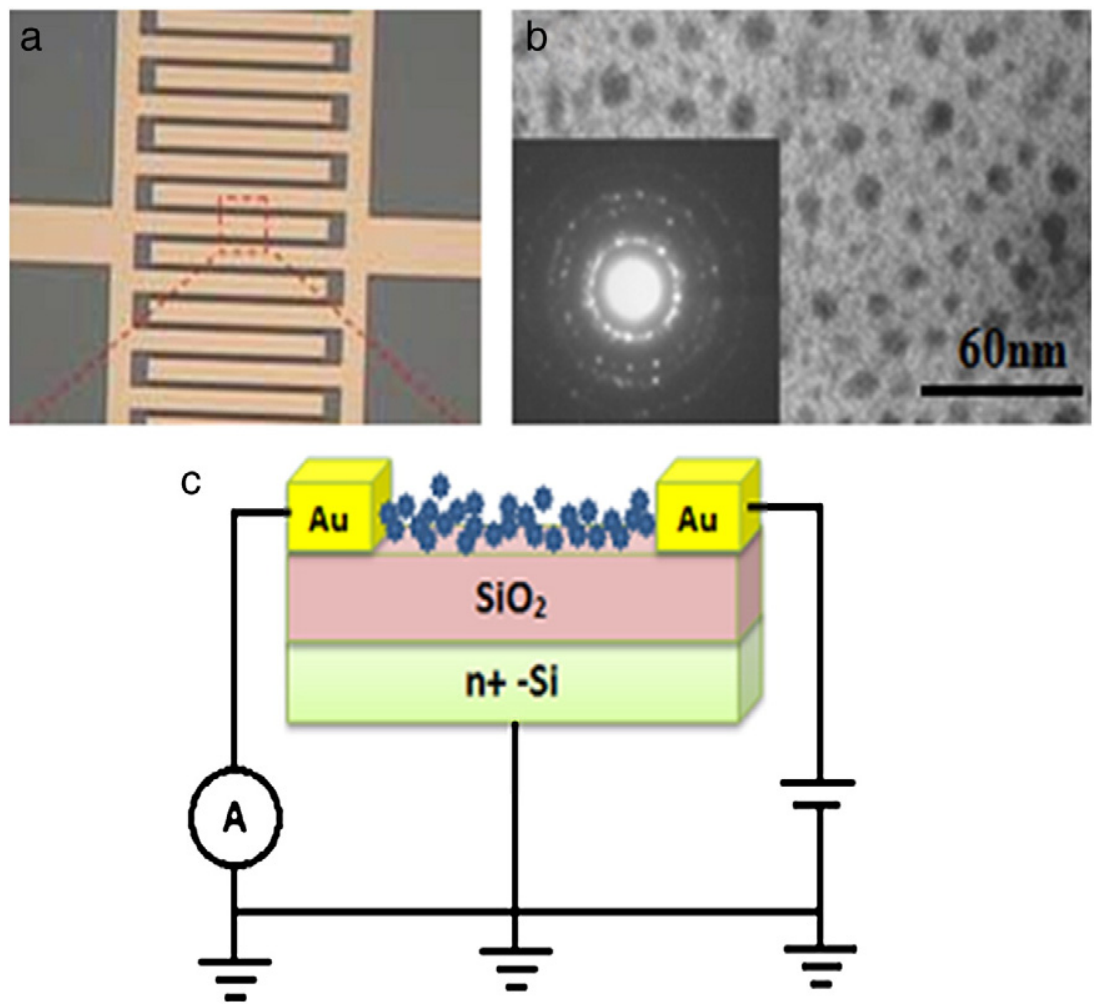

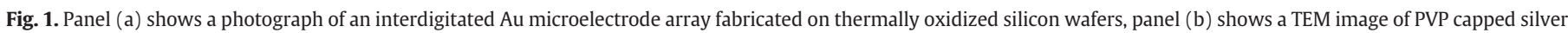
nanoparticles, and panel (c) shows a schematic diagram of the device structure associated with the measuring circuit. 

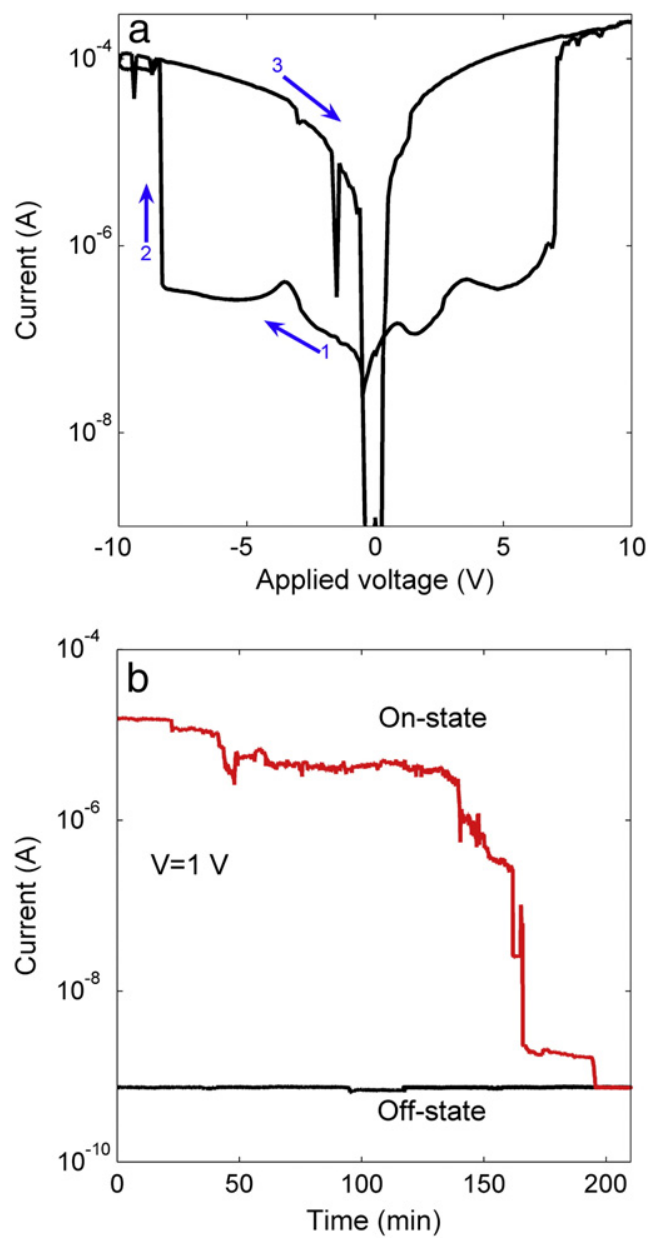

Fig. 2. Panel (a) shows the $I-V$ characteristics of a planar diode measured in air. The hysteresis loop is referred to as S-shape. At a negative voltage of $-9 \mathrm{~V}$ a sudden increase in current occurs at the high conductance state. With the voltage scanning back towards zero the diode remains in the on-state down to near $0 \mathrm{~V}$ where it suddenly switches back to the low conductance state. The arrows indicate the scanning direction. Panel (b) shows the longest retention time measured under an applied bias of $1 \mathrm{~V}$.

vacuum with S-shape switching. The diodes have to be illuminated in vacuum using a blue light emitting diode (LED) $\left(\lambda_{\max }=440 \mathrm{~nm}\right)$ for approximately ten minutes. Under illumination a photoreduction of the silver oxide occurs producing silver atoms and species of $\mathrm{Ag}_{2}^{+}$,

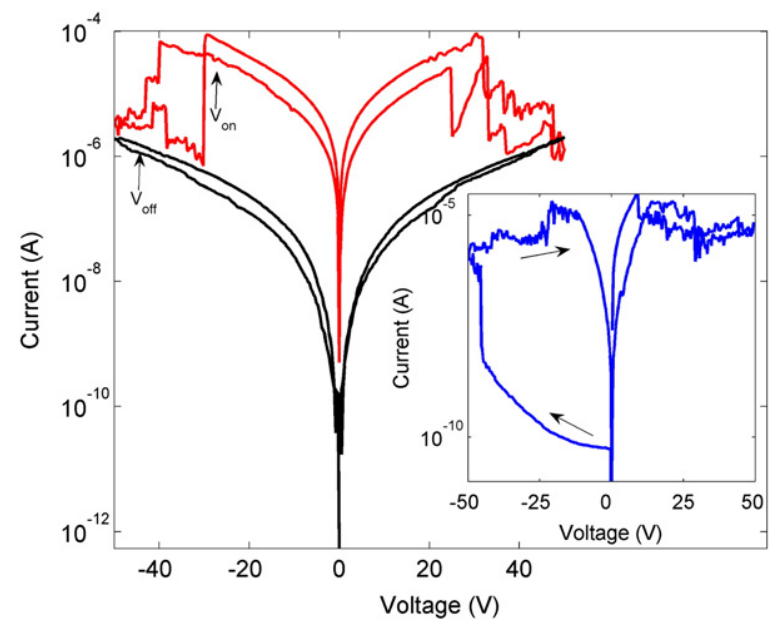

Fig. 3. Symmetric $I-V$ characteristics measured under a pressure lower than $0.01 \mathrm{~Pa}$. The hysteresis loop exhibits a pronounced NDR and is typically referred to as $\mathrm{N}$-shaped. The inset shows the electroforming loop for a pristine diode.
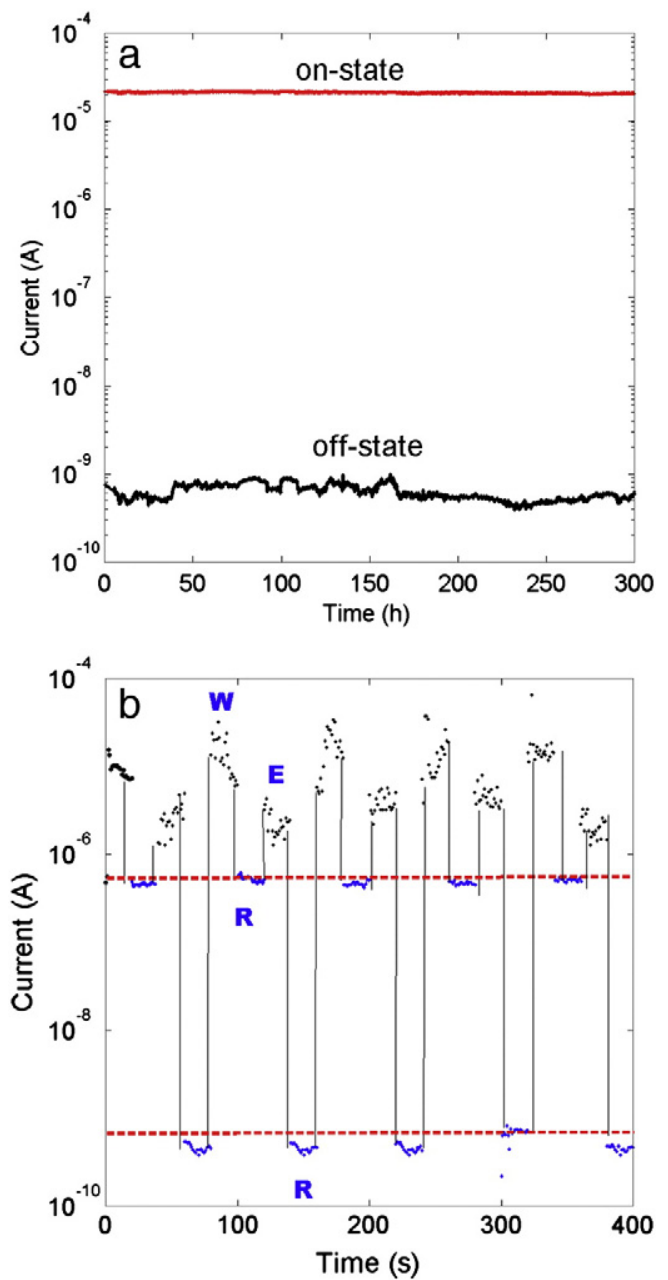

Fig. 4. Panel (a) shows the retention time measured at $3 \mathrm{~V}$ and panel (b) shows a segment part of the current response to write-read-erase-read voltage waveforms in high vacuum. The write voltage (W) is $35 \mathrm{~V}$ and the erase (E) is $50 \mathrm{~V}$. For both states the read voltage $(R)$ is $3 \mathrm{~V}$.

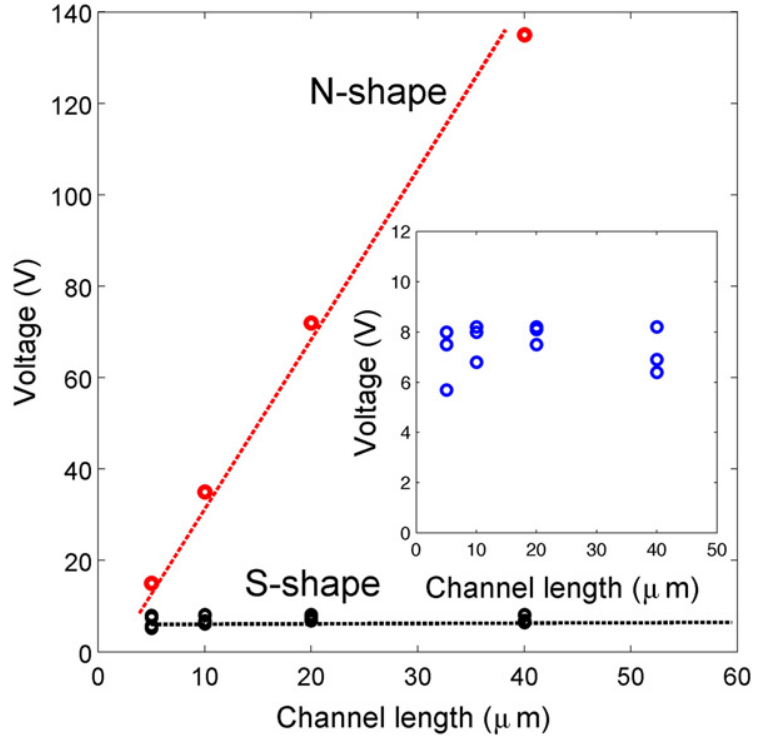

Fig. 5. Channel length dependence of the threshold voltage for switching from the off-state to the on-state for $\mathrm{N}$-shape switching and S-shape switching. 


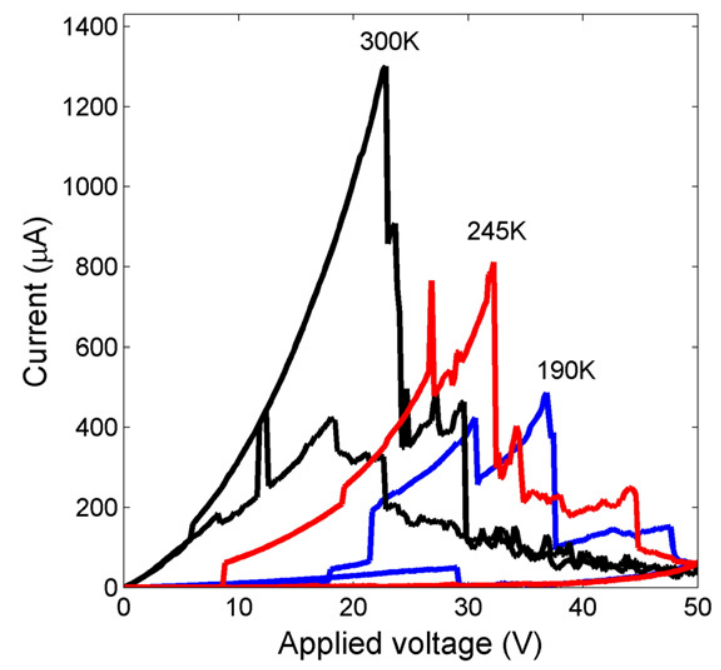

Fig. 6. $\mathrm{N}$-shaped $I-V$ curves measured for different temperatures showing that the current is thermally activated. As the sample is cooled down to $190 \mathrm{~K}$ the current decreases and the NDR region moves to higher voltages.

$\mathrm{Ag}_{3}^{+}$, and $\mathrm{Ag}_{2}$ which can be observed using fluorescence microscopy [21]. Diodes submitted to blue LED illumination show S-shape switching. Fig. 7 shows the temperature dependence of the current recorded at a fixed bias of $3 \mathrm{~V}$. The current decreases with temperature in a step-like fashion. The decrease in current is in agreement with a metallic type of conduction, suggesting that silver filaments are formed. This observation of S-shape switching is in agreement with the work of Cho et al. [22]. It also suggests that S-shape switching observed when devices are operating in air, can be caused by the water assisted electrochemical growth of $\mathrm{Ag}$ dendrites.

In order to confirm that resistive switching was caused by the presence of silver, diodes with only polymer (without silver nanoparticles) were also prepared and characterized. A typical $I-V$ characteristic of a polymer only based diode is shown in Fig. 8. As expected the polymer behaves as an insulator. No resistive switching can be observed when operated in vacuum or in air. This confirms that the polymer does not have a role in the resistive switching phenomena.

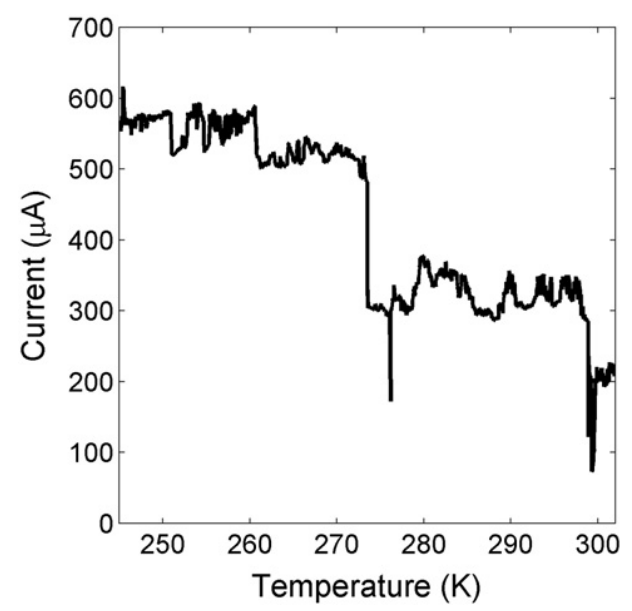

Fig. 7. Temperature dependence of the current measured at $3 \mathrm{~V}$ in a diode with $\mathrm{S}$-shape switching behavior after blue light illumination. The current increases as the sample is cooled down, and this is in agreement with a metallic type of conduction.

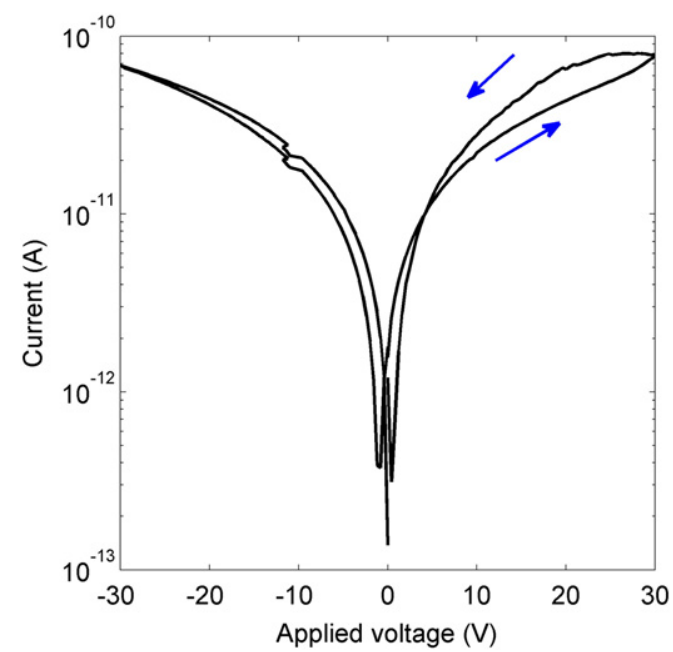

Fig. 8. I-V characteristic of a diode fabricated from a solution containing only PVP polymer (without silver nanoparticles). The behavior is typical of an insulating material with a resistance of $0.5 \times 10^{12} \Omega$ at $30 \mathrm{~V}$.

\section{Discussion}

The N-shape switching is similar to the one reported for metal/oxide based memories [23], and it requires electroforming and exhibits unipolar programming voltages. This process is irreversible as long as the device is kept in high vacuum. In our planar diodes, electroforming is not observed in air, because the diode resistance switches well below the forming voltage which is moisture mediated switching phenomena. It resembles a soft-breakdown mechanism. From our understanding of wear-out and breakdown in dielectrics, we proposed that electrons or hole tunneling (via Fowler-Nordheim or direct tunneling) create silver oxide defects. When a critical defect density is reached, the system break-downs and forms conducting paths across the nanostructured material. The microscopic nature of the defects is not known.

Switching in ambient air at low voltage (S-shape) is compatible with electrochemical reactions. It is known that water acts as an electrolyte and mediates redox reactions. Alternatively, water itself can contribute to electrical conduction. In this respect, it is relevant to know the effect of ambient atmosphere on the switching behavior. Previous works have shown that the presence of moisture is important. Tanaka [24] claimed that adsorbed water is essential to restore conducting filaments in silver thin films. Kimura et al. [25] also reported that moisture is required for resistive switching. Diffusion of hydronium ions along with silver ions was necessary to establish Ag filaments. They also showed that in vacuum switching does not occur. For encapsulated $\mathrm{Cu} / \mathrm{P} 3 \mathrm{HT} / \mathrm{Au}$ junctions, Knorr et al. [26] showed that water is field-absorbed and assists in dissolution of copper ions to form copper sulfide crystals, which may be an important precursor for filament formation. Recently, Guo et al. [27] showed that water plays a crucial role in the growth and electrochemical dissolution of Ag dendrites. Water mediates the following reaction

$\mathrm{Ag}^{+}(a q)+\mathrm{e}^{-} \rightarrow \operatorname{Ag}(\mathrm{s})$

Upon application of a voltage, $\mathrm{Ag}^{+}$ions migrate and are reduced upon contact with the electrode resulting in the growth of metallic $\mathrm{Ag}$ dendrites. The diode switches-on when a continuous percolation path along the dendrites is established. Switching-off is caused by a fast retreating of the dendrite at the neck where the dendrite contacts the electrode surface [27].

Alternatively, water itself can also contribute to electrical conduction. Surface-conductivity measurements on $\mathrm{SiO}_{2}$ suggested that 
protons $\left(\mathrm{H}^{+}\right)$that are electrolytically produced from absorbed water can act as charge carriers [28-30]. On applying a bias, oxidation of water occurs, producing excess protons in the reaction

$\mathrm{H}_{2} \mathrm{O}(\mathrm{l}) \rightarrow 2 \mathrm{H}^{+}(\mathrm{aq})+2 \mathrm{e}^{-}+1 /{ }_{2} \mathrm{O}_{2}(g)$.

In the absence of bias $\mathrm{H}^{+}$will recombine with negative species in the surrounding environment and eventually will shut-down the conducting state.

A planar diode has the conducting channel exposed, this makes it easier for atmospheric species to diffuse into the device. For the more usual sandwich type configuration, the top electrode acts as a protecting layer. However, in this case water vapor can still diffuse from the edge of the electrode into the PVP/silver oxide matrix and mediate the resistive switching mechanism. In a sandwich type of structure the diffusion of atmospheric species should then be dependent on the perimeter of the top electrode.

\section{Conclusion}

In summary, switching has been investigated in planar diodes whose conduction channels are directly exposed to the ambient atmosphere. The same diode can exhibit either an S-shape or an N-shape hysteresis loop depending on different ambient atmospheres, air/vacuum. In air, the loop is S-shaped which could be related to growth and electrochemical dissolution of Ag dendrites and/or electrical conduction mediated by water adsorbed on the surface. The on-state is volatile. In vacuum the diodes have to be electroformed, and $\mathrm{N}$-shape hysteresis loops are obtained. The origin of the N-type switching mechanism is not clear yet. We propose the formation of silver oxide species which undergo an electroforming process similar to other resistive switching oxides. When stored in vacuum, data retention and the programming cycle endurance are excellent.

\section{Acknowledgments}

We thank Ton van den Biggelaar for preparing the substrates and we gratefully acknowledge the financial support received from the European Community, Seventh Framework Program, under contract 247745, project FlexNet, from the Dutch Polymer Institute (DPI), project no. 704, and from Fundação para Ciência e Tecnologia (FCT) through the project MEMRISTOR based Adaptive Neuronal Networks (MemBrAiNN), PTDC/CTM-NAN/122868/2010.

\section{References}

[1] B. Mukherjee, A.J. Pal, Appl. Phys. Lett. 85 (2004) 2116.

[2] R.R. Sutherland, Phys. D Appl. Phys. 4 (1971) 468

[3] J. Ouyang, J. Nano Rev. 1 (2010) 5118.

[4] L.D. Bozano, B.W. Kean, M. Beinhoff, K.R. Carter, P.H. Rice, J.C. Scott, Adv. Funct. Mater. 1933 (2005) 15.

5] J.C. Scott, L.D. Bozano, Adv. Mater. 19 (2007) 1452.

[6] D. Prime, S. Paul, Phil. Trans. R. Soc. A 367 (2009) 4141.

[7] A. Kiesow, J.E. Morris, C. Radehaus, A. Heilmann, Appl. Phys. 94 (2003) 6988.

[8] H.X. Guo, B. Yang, L. Chen, Y.D. Xia, K.B. Yin, Z.G. Liu, Appl. Phys. Lett. 91 (2007) 243513.

[9] R. Muller, J. Genoe, P. Heremans, Appl. Phys. Lett. 95 (2009) 133509.

[10] T. Ouisse, O. Stephan, J. Org. Electron 5 (2004) 251.

[11] L.D. Bozano, B.W. Kean, V.R. Deline, J.R. Salem, J.C. Scot, Appl. Phys. Lett. 84 (2004) 607.

[12] P. Dimitrakis, P. Normand, D. Tsoukalas, C. Pearson, J.H. Ah, M.F. Mabrook, D.A. Zeze, M.C. Petty, K.T. Kamtekar, C. Wang, M.R. Bryce, M. Green, Appl. Phys. 104 (2008) 044510.

[13] H. Houili, E. Tutis, R. Izquierdo, Org. Electron. 11 (2010) 514

[14] J. Ouyan, Y. Yang, Appl. Phys. Lett. 96 (2010) 063506.

[15] T. Tsukamoto, S.H. Liu, Z.A. Bao, Jpn. Appl. Phys. 46 (2007) 3625.

[16] L. Goux, J.G. Lisoni, M. Jurczak, D.J. Wouters, L. Courtade, J. Appl. Phys. 107 (2010) 024512.

[17] Y.T. Tsai, T.C. Chang, W.L. Huang, C.W. Huang, Y.E. Syu, Appl. Phys. Lett. 99 (2011) 092106.

[18] K.P. Biju, X. Liu, S. Kim, S. Jung, J. Park, H. Hwang, Phys. Status Solidi RRL 5 (3) (2011) 89.

[19] L. Goux, Y.Y. Chen, L. Pantisano, X.P. Wang, G. Groeseneken, M. Jurczak, D.J. Wouters, Electrochem. Solid-State Lett. 13 (2010) 54C.

[20] H.C. Tseng, T.C. Chang, J.J. Huang, Y.T. Chen, P.C. Yang, H.C. Huang, D.S. Gan, N.J. Ho, S.M. Sze, M.J. Tsai, Thin Solid Films 520 (2011) 1656.

[21] T.H. Lee, J.I. Gonzalez, J. Zheng, R.M. Dickson, Acc. Chem. Res. 38 (2005) 534

[22] B. Cho, J.M. Yun, S. Song, Y. Ji, D.Y. Kim, T. Lee, Adv. Funct. Mater. 21 (2011) 3976.

[23] J. Beynon, J. Li, Mater. Sci. Lett. 9 (1990) 1243.

[24] F. Verbakel, S.C.J. Meskers, R.A.J. Janssen, H.L. Gomes, M. Cölle, M. Büchel, L.M. de Dago, Appl. Phys. Lett. 91 (2007) 192103.

[25] K. Tanaka, Electron. Commun. Jpn. 67-C (1984) 1.

[26] T. Kimura, K. Yamamoto, S. Yugo, Appl. Phys. Lett. 33 (1978) 15

[27] N. Knorr, R. Wirtz, S. Rosselli, G. Nelles, J. Phys. Chem. C 114 (2010) 15791.

[28] X. Guo, C. Schindler, S. Menzel, R. Waser, Appl. Phys. Lett. 91 (2007) 133513.

[29] A. Soffer, M. Folman, Trans. Faraday Soc. 62 (1966) 3559.

[30] J.H. Anderson, P.A. Parks, J. Phys. Chem. 72 (1968) 3662 